\section{Dirac's continuous creation cosmology and the temperature of the Earth}

ROXBURGH ${ }^{1}$ has contended that the present form of Dirac cosmology ${ }^{2}$ predicts too low a temperature for the Earth (in the recent past) to be acceptable. $\mathrm{He}$ computed the time dependence of the temperature of the Earth with the relation

$$
T=\left(\frac{L}{R^{2}}\right)^{1 / 4}
$$

The solar luminosity $L \sim G^{7} \times M_{\odot}{ }^{5} \sim t^{3}$, when the Dirac prescriptions $G \sim t^{-1}$, $M_{\odot} \sim t^{2}$ are used. For the time dependence of the radius of the Earth $R$ in atomic units, Roxburgh proposed and solved the equations of motion ( $(4)$ and $(4 a)$ in ref. $1)$. These equations are not valid in atomic units. Being the standard Newtonian equations, they are valid only in Einstein units where, since $m$ is constant, the differentiation between (4) and (4a) in ref. 1 is meaningless. From Roxburgh's equations, we conclude that the orbital radius in Einstein units $\bar{R}=$ constant.

Dirac $^{1}$ has argued that with multiplicative creation, $R \sim \bar{R} t$. Equation (1) then yields $T \sim t^{1 / 4}$, as shown by Canuto and Lodenquai ${ }^{3}$. Such a time variation cannot be ruled out. While Roxburgh considers Dirac's argument unconvincing, we point out that it is substantiated by dynamic equations of a consistent theory (refs. 4 and 5). In the following, we indicate four equivalent methods by which the correct equation of motion in atomic units can be derived.

(1), With a general transformation of units of the form $\mathrm{d} \bar{s}=\beta \mathrm{d} s$, where $\mathrm{d} \bar{s}$ and $\mathrm{d} s$ are the space-time intervals in Einstein and atomic units respectively, the geodesic equation of general relativity transforms into

$$
\frac{\mathrm{d}}{\mathrm{d} s}\left(\beta v^{\mu}\right): \beta \Gamma_{r \lambda}{ }^{\mu} v^{r} v^{\lambda}=g^{\mu r} \beta_{r}
$$

(2) The gravitational field equations in atomic units given in refs 4 and 5 can be used to derive

$$
T_{\mu, v^{r}}{ }^{r}=-3 \frac{\beta, v}{\beta}\left(T_{\mu}^{v}-\frac{1}{3} T_{\lambda}{ }^{2} \delta_{\mu}{ }^{v}\right)
$$

Using the stress-energy tensor of a pressureless fluid $T^{\mu r} \rho v^{\mu} \mathrm{v}^{\prime \prime}$, equation (3) reduces to the generalised geodesic equation (2).

(3) in a gauge covariant theory of gravitation $^{4}$, the tensor equations in general relativity are replaced by co-tensor equations. The geodesic equation is replaced by

$$
v^{\prime r} v_{* r}^{\mu}=0
$$

where $v^{\mu}$ is now a co-vector of power -1 and $*$ indicates co-covariant differentiation. It can be shown that equation (4) is a compact form of equation (2).

(4) Dirac ${ }^{5}$ has given an action principle from which equation (2) can be derived.

With spherical symmetry, the Newtonian limit of equation (2) gives

$$
\frac{\mathrm{d}}{\mathrm{d} t}(\beta \mathbf{r})=-\beta \frac{G M_{\odot}}{r^{2}} \mathbf{r}
$$

This equation admits the angular momentum integral

$$
\beta r^{2} \theta=h=\text { constant }
$$

and therefore the reciprocal radius $u$ satisfies the orbit equation

$$
\frac{\mathrm{d}^{2} u}{\mathrm{~d} \theta^{2}} \quad u=\beta^{2} \frac{G M}{h^{2}}
$$

Thus, the orbital radius $R$ is given by

$$
R \sim \frac{h^{2}}{G M_{\odot} \beta^{2}}
$$

In the gauge covariant theory with multiplicative creation as suggested by Dirac, it can be shown that $\beta \sim t^{-1}$. The result $R \sim t$ is thus confirmed.

There is abundant evidence to favour multiplicative creation over additive creation $^{3}$. The gauge covariant theory is consistent with co-moving multiplicative creation. Roxburgh's suggestion of multiplicative creation at rest does not have a sound theoretical framework and his equation of motion for this case seems to be an ad hoc postulate, the consequences of which ought not to be associated with Dirac cosmology.

$$
\begin{aligned}
& \text { V. Canuto } \\
& \text { P. J. Adams } \\
& \text { S. H. Hsien } \\
& \text { E. TSiang }
\end{aligned}
$$

Instutute for Space Studies, NASA, Goddard Space Flight Center, 2880 Broadway,

New York, New York 10025

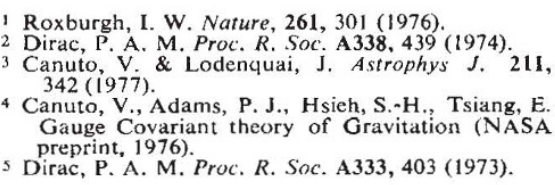

RoXbuRGH REPLIES - Canuto et al. ${ }^{1}$ correctly point out that if the equations of motion in the Dirac ${ }^{2}$ cosmology are Einstein's equations in Einstein units rather than in atomic units as I assumed ${ }^{3}$, then the orbit of the Earth around the Sun increases in time $R_{A} \propto t$, so that the temperature on the Earth increases only slowly $T \propto t^{1 / 4}$. This is only the case if matter is created at rest relative to local matter, not if it is created at rest relative to the local cosmological rest frame. In this latter case, even if the equation of motion is Einstein's in Einstein units, we have for a system of $N(\tau)$ particles of mass $m(\tau)$

$$
\frac{\mathrm{d}}{\mathrm{d} \tau}(N m v)+N M v \frac{1}{N} \frac{\mathrm{d} N}{\mathrm{~d} \tau}=-\frac{G M N m}{r^{2}{ }_{E}}
$$

and with $\mathrm{Nm}=$ constant in Einstein units this gives

$$
\frac{\mathrm{d}}{\mathrm{d} \tau}(N v)=-\frac{G M N}{r^{2}{ }_{E}}
$$

and hence $R_{E} \propto 1 / N^{2}$. In atomic units $R_{A} \sim t r_{E} \propto t / N^{2} \propto 1 / t^{3}$ so the equilibrium temperature of the Earth satisfies

$$
T_{E} \propto\left(\frac{L}{R_{A}}\right)^{1 / 4} \propto\left(\frac{G^{7} M^{5}}{R^{2}{ }_{A}}\right)^{1 / 4} \propto t^{9 / 4}
$$

which predicts a temperature of about $180 \mathrm{~K}$ when the Earth was formed.

The assumption that Einstein's equations are valid in Einstein units, however, does not uniquely predict that the creation rate $\propto t^{2}$ or that the scale factor in cosmology is $S(t) \propto t$ in atomic units as in the Dirac model. Indeed, if we demand that there be no cosmological constant in Einstein units then one solution is clearly the Einstein de Sitter Universe with $S(t) \propto t^{2 / 3}$ in Einstein units. This is satisfied with the creation rate such that $N^{\circ}(\tau) \propto \tau^{n}$ for any $n$ (my work, to be published). In atomic units this gives a scale factor

$$
S(t) \propto t^{\frac{(3 n+1)}{(3(n+1))}}
$$

For $n=0$ this is the classical Dirac cosmology $S \sim t^{1 / 3}$. The equilibrium temperature on the earth for this model then satisfies

$$
T_{E} \propto t^{\frac{(n-5)}{(4(n+1))}}
$$

and is constant for $n=: 5$. The point is that just to demand that Einstein's equations be valid in units in which $m N, G$, are constant does not determine the model, a whole family of models are possible that satisfy the large number hypothesis and Einstein's equations in suitable units, only if we demand that in atomic units $S(t) \sim t$, or equivalently that in Einstein units we have a static Einstein Universe, as we reproduce the Dirac model.

Department of Applied Mathematics, Queen Mary College,

London, UK

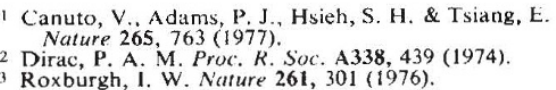

